\title{
Decay Property for Solutions to Plate Type Equations with Variable Coefficients
}

\author{
Shikuan Mao \\ School of Mathematics and Physics, \\ North China Electric Power University, \\ Beijing 102206, China
}

Xiaolu Li

School of Mathematics and Physics, North China Electric Power University, Beijing 102206, China

\begin{abstract}
In this paper we consider the initial value problem for a plate type equation with variable coefficients and memory in $\square^{n}(n \geq 1)$ ), which is of regularity-loss property. By using spectrally resolution, we study the pointwise estimates in the spectral space of the fundamental solution to the corresponding linear problem. Appealing to this pointwise estimates, we obtain the global existence and the decay estimates of solutions to the semilinear problem by employing the fixed point theorem.
\end{abstract}

Keywords: plate equation, memory, decay, regularity-loss property.

\section{INTRODUCTION}

In this paper we consider the following initial value problem for a plate type equation in $\square^{n}(n \geq 1)$ :

$\left\{\begin{array}{c}\left(1-\Delta_{g}\right) u_{t t}+\left(1+\Delta_{g}^{2}\right) u+\alpha u_{t}+\beta k *\left(-\Delta_{g}\right)^{p} u_{t}=f\left(u, u_{t}, \nabla u\right)(1.1) \\ u(x, 0)=u_{0}(x), u_{t}(x, 0)=u_{1}(x)\end{array}\right.$

Here $0 \leq p \leq 2, \alpha>0, \beta>0$ are real numbers, the subscript $t$ in $u_{t}$ and $u_{t t}$ denotes the time derivative (i.e., $\left.u_{t}=\partial_{t} u, u_{t t}=\partial_{t}^{2} u\right)$

$\Delta_{g}=\frac{1}{\sqrt{G}} \sum_{i j=1}^{n} \partial_{x_{i}} \sqrt{G} g^{i j} \partial_{x_{j}}$ is the Laplace(-Beltrami) operator associated with the Riemannian metric

$g=\sum_{i j=1}^{n} g_{i j}(x) d x_{i} \otimes d x_{j}, G=\left|\operatorname{det}\left(g_{i j}\right)\right|$

and $\left(g^{i j}\right)=\left(g_{i j}\right)^{-1}, u=u(x, t)$ is the unknown function of $x \in R^{n}$ and $t>0$, and represents the transversal displacement of the plate at the point $x$ and $t, \Delta_{g} u_{t t}$ corresponds to the rotational inertial. The term $u_{t}$ represents a frictional dissipation to the plate. The term

$k *\left(-\Delta_{g}\right)^{p} u_{t}:=\int_{0}^{t} k(t-\tau)\left(-\Delta_{g}\right)^{p} u_{t}(\tau) d \tau$

corresponds to the memory term, and $k(t)$ satisfies the following assumptions:

Assumption [A]. $k \in C^{2}\left(R^{+}\right), k(s)>0$, and the derivatives of $k$ satisfy the following conditions

$$
-C_{0} k(s) \leq k^{\prime}(s) \leq-C_{1} k(s),
$$

$$
C_{2} k(s) \leq k^{\prime \prime}(s) \leq C_{3} k(s), \forall s \in\left(R^{+}\right)
$$

Where $C_{i}(i=0,1,2,3)$ are positive constants.

We suppose the metric $g$ satisfies the following conditions:

Assumption [B]. The matrix $g^{i j}$ is symmetric for each $x \in \square^{n}$, and there exists $C_{\alpha}>0$ and $C>0$ such that

(i) $g^{i j} \in C^{\infty}\left(\square^{n}\right),\left|\partial_{x}^{\alpha} g^{i j}(x)\right| \leq C_{\alpha}$,

$\forall x \in \square^{n}, \alpha \in \square_{+}^{n}$.

(ii) $C_{1}|\xi|^{2} \leq \sum_{i j=1}^{n} g^{i j}(x) \xi_{i} \xi_{j} \leq C_{2}|\xi|^{2}$,

$\forall x \in \square^{n}, \forall \xi \in \square^{n}$.

Assumption [C]. $f \in C^{\infty}\left(\square^{n+2}\right)$ and there exists $\eta \in \square^{+}$ satisfying $\eta>1$ such that $f(U)=O\left(|U|^{n}\right)$, as $|U| \rightarrow 0$.

It is well known that under the above assumptions, the Laplace operator $\Delta_{g}$ is essentially self-adjoint on the Hilbert $e^{t \Delta_{g}}$ space $H=L^{2}\left(\square^{n}, d \mu_{g}\right)$ with domain $C_{0}^{\infty}\left(\square^{n}\right)$, here $d \mu_{g}=\sqrt{G} d x$. We denote the unique self-adjoint extension (to the Sobolev space $H^{2}\left(\square^{n}\right)$ ) by the same symbol $\Delta_{g}$. The spectrum of $-\Delta_{g}$ is $[0,+\infty)$, and it generates a contraction semi-group $e^{t \Delta_{g}}$ on $L^{p}\left(\square^{n}\right)$ 
$(1 \leq p \leq \infty)$, We note by our assumptions of the metric $g$, the measure $d \mu_{g}$ is equivalent to $d x$, and by the (functional) calculus for pseudodifferential operators, we have the following classical equivalence (cf. $[8,5]$ ): For $S \in \square$, there exists $C_{s}>0$, such that

$$
\begin{aligned}
C_{s}^{-1}\|u\|_{H^{s}} \leq\left(u,\left(1-\Delta_{g}\right)^{\frac{s}{2}} u\right)_{L^{2}} & \leq C_{s}\|u\|_{H^{s}} \\
\forall u & \in S\left(\square^{n}\right)
\end{aligned}
$$

where $S\left(\square^{n}\right)$ is the class of Schwartz functions.

The main purpose of this paper is to study the global existence and decay estimates of solutions to the initial value problem (1.1). For our problem, it is difficult to obtain explicitly the solution operators or their Fourier transform due to the presence of the memory term and variable coefficients. However, we can obtain the pointwise estimate in the spectral space of the fundamental solution operators to the corresponding linear equation

$$
\begin{array}{r}
\left(1-\Delta_{g}\right) u_{t t}+\left(1+\Delta_{g}^{2}\right) u+\alpha u_{t} \\
+\beta k *\left(-\Delta_{g}\right)^{p} u_{t}=0
\end{array}
$$

from which the global existence and the decay estimates of solutions to the semilinear problem can be obtained. The following are our main theorems.

Theorem 1.1 (energy estimate for linear problem). Let $S>0$ be a real number. Assume that $u_{0} \in H^{s+\max \{1,2 p-2\}}\left(\square^{n}\right)$

and $u_{1} \in H^{s}\left(\square^{n}\right)$, and put

$$
I_{0}=\left\|u_{0}\right\|_{H^{s+\max \{1,2 p-2\}}}+\left\|u_{1}\right\|_{H^{s}} .
$$

Then the solution to the problem (1.3) with initial condition $u(0)=u_{0}$ and $u_{t}(0)=u_{1}$ satisfies

$$
u \in C^{0}\left([0, \infty) ; H^{s+1}\left(\square^{n}\right)\right) \cap C^{1}\left([0, \infty) ; H^{s}\left(\square^{n}\right)\right)
$$

and the following energy estimate:

$$
\begin{aligned}
\|u(t)\|_{H^{s+1}}^{2}+ & \left\|u_{t}(t)\right\|_{H^{s}}^{2} \\
& +\int_{0}^{t}\left(\|u(\tau)\|_{H^{s}}^{2}+\left\|u_{t}(\tau)\right\|_{H^{s-1}}^{2}\right) d \tau \leq C I_{0}^{2} .
\end{aligned}
$$

The second one is about the decay estimates for the solution to (1.3), which is stated as follows:

Theorem 1.2 (decay estimates for linear problem). Under the same assumptions as in Theorem 1.1, then the solution to (1.3) satisfies the following decay estimates:

$$
\|u(t)\|_{H^{s+1-\sigma}} \leq C I_{0}(1+t)^{-\frac{\sigma}{2}}
$$

for $0 \leq \sigma \leq s+1$, and

$$
\left\|u_{t}(t)\right\|_{H^{s-\sigma}} \leq C I_{0}(1+t)^{-\frac{\sigma}{2}}
$$

for $0 \leq \sigma \leq s$.

Theorem 1.3 (existence and decay estimates for semilinear problem). Let $s>\frac{n}{2}$ and $0 \leq p \leq 2$ be real numbers. Assume that $u_{0} \in H^{S+\max \{1,2 p-2\}}\left(\square^{n}\right), u_{1} \in H^{s}\left(\square^{n}\right)$, and put

$$
I_{0}=\left\|u_{0}\right\|_{H^{s+\max \{1,2 p-2\}}}+\left\|u_{1}\right\|_{H^{s}}
$$

then there exists a small $\varepsilon>0$, such that when $I_{0} \leq \varepsilon$, there exists a unique solution to (1.1) in

$u \in C^{0}\left([0, \infty) ; H^{s+1}\left(\square^{n}\right)\right) \cap C^{1}\left([0, \infty) ; H^{s}\left(\square^{n}\right)\right)$ satisfying the following decay estimates:

$$
\|u(t)\|_{H^{s+1-\sigma}} \leq C I_{0}(1+t)^{-\frac{\sigma}{2}},
$$

for $0 \leq \sigma \leq s+1$, and

$$
\left\|u_{t}(t)\right\|_{H^{s-\sigma}} \leq C I_{0}(1+t)^{-\frac{\sigma}{2}},
$$

for $0 \leq \sigma \leq s$.

Remark 1. If the semilinear term is the form of $f(u)$, then we may assume $s>0(n=1)$ and $s+1 \geq \frac{n}{2}(n \geq 2)$ in Theorem 1.3.

For the study of plate type equations, there are many results in the literatures. In [4], da Luz-Charão studied a semilinear damped plate equation :

$$
u_{t t}-\Delta u_{t t}+\Delta^{2} u+u_{t}=f(u)
$$

They proved the global existence of solutions and a polynomial decay of the energy by exploiting an energy method. However the result was restricted to dimension $1 \leq n \leq 5$, This restriction on the space dimension was removed by Sugitani-Kawashima (see [23]) by the fundamental method of energy estimates in the Fourier (or frequency) space and some sharp decay estimates. Since the method of energy estimates in Fourier space is relatively simple and effective, it has been adapted to study some related problems (see [18, 19, 20, 24]).

For the case of dissipative plate equations of memory type, Liu-Kawashima (see [15, 12]) studied the following equation

$$
u_{t t}+\Delta^{2} u+u+k * \Delta u=f(u)
$$


as well as the equation with rotational term

$$
u_{t t}-\Delta u_{t t}+\Delta^{2} u+u+k * \Delta u=f\left(u, u_{t}, \nabla u\right),
$$

and obtained the global existence and decay estimates of solutions by the energy method in the Fourier space. The results in these papers and the general dissipative plate equation (see $[13,14,16,23]$ ) show that they are of regularity-loss property.

A similar decay structure of the regularity-loss type was also observed for the dissipative Timoshenko system (see [10]) and a hyperbolic-elliptic system related to a radiating gas (see [9]). For more studies on various aspects of dissipation of plate equations, we refer to $[1,2,3,7]$. And for the study of decay properties for hyperbolic systems of memory-type dissipation, we refer to $[6,11,22]$.

The results in [12] are further studied and generalized to higher order equations in [16] and to the equations with variable coefficients in [17]. The main purpose of this paper is to study the decay estimates and regularity-loss property for solutions to the initial value problem (1.1) in the spirit of [12, $15,16,17]$. And we generalize these results to the case of variable coefficients and semilinear equations.

The paper is arranged as follows: We study the pointwise estimates of solutions to the problem (2.2) and (2.3) in the spectral space in Section 2. And in Section 3, we prove the energy estimates and the decay estimates for solutions to the linear equation (1.3) by virtue of the estimates in Section 2. In Section 4, we prove the global existence and decay estimates for the semilinear problems (1.1).

For the reader's convenience, we give some notations which will be used below. Let $F[f]$ denote the Fourier transform of $f$ defined by

$$
F[f]=\hat{f}(\xi):=\frac{1}{(2 \pi)^{\frac{n}{2}}} \int_{R^{n}} e^{-i x \xi} f(x) d x
$$

and we denote its inverse transform as $F^{-1}$.

Let $L[f]$ denote the Laplace transform of $f$ defined by

$$
\|f\|_{H^{s}}=\left\|(1-\Delta)^{\frac{s}{2}} f\right\|_{L^{2}\left(R_{x}^{n}\right)} \cong\left\|\langle\xi\rangle^{s} \hat{f}\right\|_{L^{2}\left(R_{x}^{n}\right)}
$$

here $\langle\xi\rangle=\left(1+|\xi|^{2}\right)^{\frac{1}{2}}$ denotes the Japanese bracket.

\section{Pointwise estimates in the spectral space.}

We observe that the equation (1.1) ( respectively (1.3) ) is equivalent to the following in-homeogeneous equation

$$
\begin{aligned}
& \left(1-\Delta_{g}\right) u_{t t}+\left(1+\Delta_{g}^{2}\right) u+\beta k(0)\left(-\Delta_{g}\right)^{p} u \\
& +\alpha u_{t}+\beta k^{\prime} *\left(-\Delta_{g}\right)^{p} u=F(t, x) \\
& \text { with } F(t, x)=k(t)\left(-\Delta_{g}\right)^{p} u_{0}(x)+f\left(u, u_{t}, \nabla u\right) \\
& \text { (respectively, } F(t, x)=k(t)\left(-\Delta_{g}\right)^{p} u_{0}(x) \text { ). }
\end{aligned}
$$

In order to study the solutions to (2.1), we study the pointwise estimates for solutions to the following ODEs with parameter $\lambda \in \square^{+}$, respectively:

$$
\left\{\begin{array}{c}
\left(1+\lambda^{2}\right) G_{t t}(t, \lambda)+\left(1+\lambda^{4}+\beta k(0) \lambda^{2 p}\right) G(t, \lambda) \\
+\alpha G_{t}(t, \lambda)+\beta \lambda^{2 p} k^{\prime} * G=0 \\
G(0, \lambda)=1, G_{t}(0, \lambda)=0,
\end{array}\right.
$$

and

$$
\left\{\begin{array}{l}
\left(1+\lambda^{2}\right) H_{t t}(t, \lambda)+\left(1+\lambda^{4}+\beta k(0) \lambda^{2 p}\right) H(t, \lambda) \\
\quad+\alpha H_{t}(t, \lambda)+\beta \lambda^{2 p} k^{\prime} * H=0 \\
H(0, \lambda)=0, H_{t}(0, \lambda)=1,
\end{array}\right.
$$

We note that $G(t, \lambda)=H_{t}(t, \lambda)+\langle\lambda\rangle^{-2} H(t, \lambda)$, and apply the Laplace transform to (2.2) and (2.3) (which is guaranteed by Proposition 1 given at the end of this section), then we have formally that

$G(t, \lambda)=L_{\tau \rightarrow t}^{-1}\left[\frac{\alpha+\left(1+\lambda^{2}\right) \tau}{\left(1+\lambda^{2}\right) \tau^{2}+\left(1+\lambda^{4}+\beta k(0) \lambda^{2 p}\right)+\alpha \tau+\beta \lambda^{2 p} L\left[k^{\prime}\right](\tau)}\right](t)$

$H(t, \lambda)=L_{\tau \rightarrow t}^{-1}\left[\frac{\left(1+\lambda^{2}\right)}{\left(1+\lambda^{2}\right) \tau^{2}+\left(1+\lambda^{4}+\beta k(0) \lambda^{2 p}\right)+\alpha+\beta \lambda^{2 p} L\left[k^{\prime}\right](\tau)}\right](t)$

Now by virtue of the solutions to (2.2) and (2.3), the solution to (2.1) can be expressed as

$$
\begin{aligned}
u(t) & =G(t, \Lambda) u_{0}+H(t, \Lambda) u_{1} \\
& +\int_{0}^{t} H(t-\tau, \Lambda)\left(1-\Delta_{g}\right)^{-1} F(\tau) d \tau
\end{aligned}
$$

where $G(t, \Lambda)$ and $H(t, \Lambda)$ ) are defined by the measurable functional calculus (cf. [21]):

$$
\left\{\begin{array}{l}
(\varphi, \quad G(t, \Lambda) \psi)=\int_{R} G(t, \lambda) d\left(P_{\lambda} \varphi, \psi\right)_{L^{2}} \\
(\varphi, H(t, \Lambda) \psi)=\int_{R} H(t, \lambda) d\left(P_{\lambda} \varphi, \psi\right)_{L^{2}}
\end{array}\right.
$$

for $\varphi, \psi$ in the domain of $G(t, \Lambda)$ and $H(t, \Lambda)$ 
respectively, here $\left\{P_{\lambda}\right\}$ is the family of spectral projections for the positive self-adjoint operator $\Lambda=\left(-\Delta_{g}\right)^{\frac{1}{2}}$. We note that $G(t, \Lambda)$ and $H(t, \Lambda)$ are the solutions (formally) to the following operator equations:

$$
\left\{\begin{aligned}
&\left(1-\Delta_{g}\right) G_{t t}+\left(1+\Delta_{g}^{2}+\beta k(0)\left(-\Delta_{g}^{p}\right)\right) G \\
&+\alpha G_{t}+\beta k^{\prime} *\left(-\Delta_{g}\right)^{p} G=0 \\
& G(0)=I, G_{t}(0)=O
\end{aligned}\right.
$$

and

$$
\left\{\begin{aligned}
&\left(1-\Delta_{g}\right) H_{t t}+\left(1+\Delta_{g}^{2}+\beta k(0)\left(-\Delta_{g}^{p}\right)\right) H \\
&+\alpha H_{t}+\beta k^{\prime} *\left(-\Delta_{g}\right)^{p} H=0 \\
& H(0)=O, H_{t}(0)=I
\end{aligned}\right.
$$

respectively, here $I$ stands for the identity operator, and $O$ denotes the zero operator

Thus estimates for $u(t)$ can be reduced to estimates for $G(t, \Lambda)$ and $H(t, \Lambda)$ in terms of (2.4).

First, let us introduce some notations. For any reasonable complex-valued function $f(t), t \in[0, \infty)$, we define

$$
\begin{aligned}
& (k * f)(t):=\int_{0}^{t} k(t-\tau) f(\tau) d \tau, \\
& (k \diamond f)(t):=\int_{0}^{t} k(t-\tau)(f(\tau)-f(t)) d \tau, \\
& (k \square f)(t):=\int_{0}^{t} k(t-\tau)|f(t)-f(\tau)|^{2} d \tau .
\end{aligned}
$$

Then direct computations imply the following lemma

Lemma 2.1. For any functions $k \in C^{1}\left(R^{+}\right)$and $\phi \in H^{1}\left(R^{+}\right)$, it holds that

(1). $\quad(k * \phi)(t)=(k \diamond \phi)(t)+\left(\int_{0}^{t} k(\tau) d \tau\right) \phi(t)$,

(2). $\operatorname{Re}\left\{(k * \phi)(t) \bar{\phi}_{t}(t)\right\}=-\frac{1}{2} k(t)|\phi(t)|^{2}$ $+\frac{1}{2}(k \sqcup \phi)(t)-\frac{1}{2} \frac{d}{d t}\left\{(k \square \phi)(t)-\left(\int_{0}^{t} k(\tau) d \tau\right)|\phi(t)|^{2}\right\}$

(3). $\quad|(k \diamond \phi)(t)|^{2} \leq\left(\int_{0}^{t}|k(\tau)| d \tau\right)(|k| \phi)(t)$.
Remark 2. From Lemma 2.1 1), we have

$$
(k * \phi)_{t}=k(0) \phi+k^{\prime} * \phi=k(t) \phi+k^{\prime} \diamond \phi
$$

Now we come to get the pointwise estimates of $G(t, \lambda)$ and $H(t, \lambda)$ in the spectral space, and we have the following proposition.

Proposition 1 (pointwise estimates in the spectral space). Assume $G(t, \lambda)$ and $H(t, \lambda)$ are the solutions of (2.2) and (2.3) respectively, then they satisfy the following estimates:

$$
\begin{aligned}
\left|\langle\lambda\rangle G_{t}(t, \lambda)\right|^{2} & +\left|\langle\lambda\rangle^{2} G(t, \lambda)\right|^{2} \\
& +\beta|\lambda|^{2 p}(k \square G)(t, \lambda) \leq C e^{-c \rho(\lambda) t}\langle\lambda\rangle^{4},
\end{aligned}
$$

And

$$
\begin{aligned}
\left|\langle\lambda\rangle H_{t}(t, \lambda)\right|^{2} & +\left|\langle\lambda\rangle^{2} H(t, \lambda)\right|^{2} \\
& +\beta|\lambda|^{2 p}(k \square H)(t, \lambda) \leq C e^{-c \rho(\lambda) t}\langle\lambda\rangle^{4},
\end{aligned}
$$

here $k \square G$ and $k \square H$ are defined as in (2.8), and

$$
\rho(\lambda)=\langle\lambda\rangle^{-2} \text { with }\langle\lambda\rangle=\left(1+\lambda^{2}\right)^{\frac{1}{2}}
$$

Proof. We only prove the estimate for $G(t, \lambda)$, and the case for $H(t, \lambda)$ can be proved in a similar way. To simplify the notation in the following, we write $G$ for $G(t, \lambda)$.

Step 1. By multiplying (2.2) by $\bar{G}_{t}$ and taking the real part, we have that

$$
\begin{gathered}
\left\{\frac{1}{2}\langle\lambda\rangle^{2}\left|G_{t}\right|^{2}\right\}_{t}+\left\{\frac{1}{2}\left(1+\lambda^{4}+\beta \lambda^{2 p} k(t)\right)|G|^{2}\right\} \\
+\alpha\left|G_{t}\right|^{2}+\beta \lambda^{2 p} \operatorname{Re}\left\{\left(k^{\prime} * G\right) \bar{G}_{t}\right\}=0
\end{gathered}
$$

Apply Lemma 2.12 ) to the term $\operatorname{Re}\left\{\left(k^{\prime} * G\right) \bar{G}_{t}\right\}$ in (2.9), and denote

$$
\begin{aligned}
E_{1}(t, \lambda) & :=\frac{1}{2}\left(1+\lambda^{2}\right)\left|G_{t}\right|^{2}+\frac{1}{2}\left(1+\lambda^{4}+\beta \lambda^{2 p} k(t)|G|^{2}\right) \\
& -\frac{\beta \lambda^{2 p}}{2}\left(k^{\prime} \square G\right),
\end{aligned}
$$

and 
$\left|R_{2}(t, \lambda)\right| \leq C\left(\langle\lambda\rangle^{2}\left|G_{t}\right|^{2}+\frac{\beta \lambda^{2 p}}{2} k(0)|G|^{2}\right)+\frac{\beta \lambda^{2 p}}{2}\left(k^{\prime \prime} \square G\right)$, then we obtain that

$$
\frac{\partial}{\partial t} E_{1}(t, \lambda)+F_{1}(t, \lambda)=0
$$

Step 2. By multiplying (2.2) by $\bar{G}$ and taking the real part, we have that

$$
\begin{aligned}
\operatorname{Re}\left\{\left(1+\lambda^{2}\right) G_{t} \bar{G}\right\}_{t}-\left(1+\lambda^{2}\right)\left|G_{t}\right|^{2} & \\
+(1+ & \left.\lambda^{4}+\beta k(0) \lambda^{2 p}\right)|G|^{2}+\alpha\left\{\frac{1}{2}|G|^{2}\right\}_{t} \\
+\beta \lambda^{2 p} \operatorname{Re}\left\{\left(k^{\prime} * G\right) \bar{G}\right\} & =0 .
\end{aligned}
$$

In view of Lemma 2.1 1), we have that

$$
\begin{aligned}
\operatorname{Re}\left\{\left(k^{\prime} * G\right) \bar{G}\right\} & =\operatorname{Re}\left\{\left(k^{\prime} \diamond G\right) \bar{G}\right\}+\left(\int_{0}^{t} k^{\prime}(\tau) d \tau\right) G \bar{G} \\
& =\operatorname{Re}\left\{\left(k^{\prime} \diamond G\right) \bar{G}\right\}+(k(t)-k(0))|G|^{2}
\end{aligned}
$$

Denote

$$
\begin{aligned}
& E_{2}(t, \lambda):=\operatorname{Re}\left\{\left(1+\lambda^{2}\right) G_{t} \bar{G}\right\}+\frac{\alpha}{2}|G|^{2}, \\
& F_{2}(t, \lambda):=\left(1+\lambda^{4}+\beta k(t) \lambda^{2 p}\right)|G|^{2}, \\
& R_{2}(t, \lambda):=\left(1+\lambda^{2}\right)\left|G_{t}\right|^{2}-\beta \lambda^{2 p} \operatorname{Re}\left\{\left(k^{\prime} \diamond G\right) \bar{G}\right\},
\end{aligned}
$$

then (2.11) yields that

$$
\frac{\partial}{\partial t} E_{2}(t, \lambda)+F_{2}(t, \lambda)=R_{2}(t, \lambda)
$$

Step 3. Define $\rho(\lambda)=\langle\lambda\rangle^{-2}$, and set

$$
\begin{aligned}
& E(t, \lambda):=E_{1}(t, \lambda)+\gamma \rho(\lambda) E_{2}(t, \lambda), \\
& F(t, \lambda):=F_{1}(t, \lambda)+\gamma \rho(\lambda) F_{2}(t, \lambda), \\
& R(t, \lambda):=\gamma \rho(\lambda) R_{2}(t, \lambda),
\end{aligned}
$$

Here $\gamma$ is a positive constant and will be determined later. Then (2.10) and (2.12) yields that

$$
\frac{\partial}{\partial t} E(t, \lambda)+F(t, \lambda)=R(t, \lambda)
$$

We introduce the following Lyapunov functions:

$$
\begin{aligned}
& E_{0}(t, \lambda):=\frac{1}{2}\left(1+\lambda^{2}\right)\left|G_{t}\right|^{2}+\frac{1}{2}\left(1+\lambda^{4}\right)|G|^{2}+\frac{1}{2} \beta \lambda^{2 p}(k \square G), \\
& F_{0}(t, \lambda):=\left|G_{t}\right|^{2}+\frac{\beta \lambda^{2 p}}{2} k(t)|G|^{2}+\frac{\beta \lambda^{2 p}}{2}\left(k^{\prime \prime} \square G\right) .
\end{aligned}
$$

From the definition of $E_{1}(t, \lambda)$ and $F_{1}(t, \lambda)$, we know that there exist positive constants $C_{i}(i=1,2,3)$ such that the following estimates hold:

$$
\begin{aligned}
& C_{1} E_{0}(t, \lambda) \leq E_{1}(t, \lambda) \leq C_{2} E_{0}(t, \lambda), \\
& F_{1}(t, \lambda) \geq C_{3} F_{0}(t, \lambda) .
\end{aligned}
$$

On the other hand, since

$$
\left|E_{2}(t, \lambda)\right| \leq C\left(\langle\lambda\rangle^{4}\left|G_{t}\right|^{2}+|G|^{2}\right),
$$

we know that

$$
\begin{aligned}
\gamma \rho(\lambda)\left|E_{2}(t, \lambda)\right| \leq & \gamma C\left(\langle\lambda\rangle^{2}\left|G_{t}\right|^{2}+\left(1+\lambda^{4}\right)|G|^{2}\right) \\
& +\gamma C\left(\frac{\beta \lambda^{2 p}}{2}(k \llbracket G)\right) \leq \gamma C_{4} E_{0}(t, \lambda) .
\end{aligned}
$$

Choosing $\gamma$ suitably small such that $\gamma C_{4} \leq \min \left\{\frac{C_{1}}{2}, \frac{C_{2}}{2}\right\}$, and by virtue of (2.14), we have that

$\frac{C_{1}}{2} E_{0}(t, \lambda) \leq E(t, \lambda) \leq \frac{3 C_{2}}{2} E_{0}(t, \lambda)$.

In view of (2.14), it is easy to verify that

$F(t, \lambda) \geq C_{3} F_{0}(t, \lambda)+C_{3} \gamma \rho(\lambda)\left(1+\lambda^{4}+\beta k(t) \lambda^{2 p}\right) \mid G^{2}$

Since

$$
\begin{aligned}
\left|R_{2}(t, \lambda)\right| \leq & C\left(\langle\lambda\rangle^{2}\left|G_{t}\right|^{2}+\frac{\beta \lambda^{2 p}}{2} k(0)|G|^{2}\right) \\
& +C \frac{\beta \lambda^{2 p}}{2}\left(k^{\prime \prime} \square G\right) .
\end{aligned}
$$

We have

$$
\begin{aligned}
|R(t, \lambda)| \leq & \gamma C\left(\left|G_{t}\right|^{2}+\rho\langle\lambda\rangle \frac{\beta \lambda^{2 p}}{2} k(0)|G|^{2}\right) \\
& +\gamma C \rho(\lambda) \frac{\beta \lambda^{2 p}}{2}\left(k^{\prime \prime} \square G\right) \\
\leq & \gamma C_{5} F(t, \lambda) .
\end{aligned}
$$

Taking $\gamma$ sufficiently small such that 
$\gamma \leq\left\{\frac{1}{2 C_{5}}, C_{4}^{-1} \min \left\{\frac{C_{1}}{2}, \frac{C_{2}}{2}\right\}\right\}$, we have that

$$
|R(t, \lambda)| \leq \frac{1}{2} F(t, \lambda)
$$

In view of (2.13), the relation (2.17) yields that

$$
\frac{\partial}{\partial t} E(t, \lambda)+\frac{1}{2} F(t, \lambda) \leq 0
$$

On the other hand, (2.15) and (2.16) yield that

$$
F(t, \lambda) \geq c \rho(\lambda) E(t, \lambda)
$$

Then (2.18) and (2.19) yield that

$$
E(t, \lambda) \leq e^{-c \rho(\lambda)} E(0, \lambda)
$$

By virtue of (2.15) and (2.20), we get the desired results.

\section{Decay estimates of solutions to the linear problem.}

In this section we shall use the functional calculus of $\Lambda$ and the pointwise estimates in spectral space obtained in Proposition 1 to prove the energy estimate in Theorem 1.1 and the decay estimates in Theorem 1.2.

Proof of Theorem 1.1. From (2.18) and (2.19) we have that

$$
\frac{\partial}{\partial t} E(t, \lambda)+C \rho(\lambda) E(t, \lambda) \leq 0
$$

Integrate the previous inequality with respect to $t$ and appeal to $(2.15)$, then we obtain

$$
E_{0}(t, \lambda)+\int_{0}^{t} \rho(\lambda) E_{0}(\tau, \lambda) \leq C E_{0}(t, \lambda) .
$$

Multiply (3.1) by $\langle\lambda\rangle^{2(s-1)}$ and integrate the resulting inequality with respect to the measure $d\left(P_{\lambda} u_{0}, u_{0}\right)$, as well as by the definition of $G(t, \Lambda)$ in (2.5) and the equivalence (1.2), then we obtain the following estimate for $G(t, \Lambda) u_{0}$ :

$$
\begin{aligned}
& \left\|G(t, \Lambda) u_{0}\right\|_{H^{s+1}}^{2}+\left\|G_{t}(t, \Lambda) u_{0}\right\|_{H^{s}}^{2} \\
& \quad+\int_{0}^{t}\left(\left\|G(\tau, \Lambda) u_{0}\right\|_{H^{s}}^{2}+\left\|G_{t}(\tau, \Lambda) u_{0}\right\|_{H^{s-1}}^{2}\right) d \tau \\
& \quad \leq C\left\|u_{0}\right\|_{H^{s+1}}^{2}
\end{aligned}
$$

Similarly, we have

$$
\begin{aligned}
& \left\|H(t, \Lambda) u_{1}\right\|_{H^{s+1}}^{2}+\left\|H_{t}(t, \Lambda) u_{1}\right\|_{H^{s}}^{2} \\
& \quad+\int_{0}^{t}\left(\left\|H(\tau, \Lambda) u_{1}\right\|_{H^{s}}^{2}+\left\|H_{t}(\tau, \Lambda) u_{1}\right\|_{H^{s-1}}^{2}\right) d \tau \\
& \quad \leq C\left\|u_{1}\right\|_{H^{s+1}}^{2}
\end{aligned}
$$

From (3.3), we know that

$\left\|H(t, \Lambda) u_{1}\right\|_{H^{s+1}} \leq C\left\|u_{1}\right\|_{H^{s}}, \quad \forall u_{1} \in S\left(\square^{n}\right), t>0$,

which implies

$$
\begin{aligned}
& \left\|\int_{0}^{t} k(\tau) H(\tau, \Lambda)\left(-\Delta_{g}\right)^{p}\left(1-\Delta_{g}\right)^{-1} u_{0} d \tau\right\|_{H^{s+1}} \\
& \leq \int_{0}^{t} k(\tau) d \tau \sup _{\tau \in[0, t]}\left\|H(\tau, \Lambda)\left(-\Delta_{g}\right)^{p}\left(1-\Delta_{g}\right)^{-1} u_{0}\right\|_{H^{s+1}} \\
& \leq C\left\|u_{0}\right\|_{H^{s+2 p-2}} .
\end{aligned}
$$

Similarly, we have

$$
\begin{aligned}
& \left\|\int_{0}^{t} k(\tau) H_{t}(\tau, \Lambda)\left(-\Delta_{g}\right)^{p}\left(1-\Delta_{g}\right)^{-1} u_{0} d \tau\right\|_{H^{s}} \\
& \leq \int_{0}^{t} k(\tau) d \tau \sup _{\tau \in[0, t]}\left\|H_{t}(\tau, \Lambda)\left(-\Delta_{g}\right)^{p}\left(1-\Delta_{g}\right)^{-1} u_{0}\right\|_{H^{s}} \\
& \leq C\left\|u_{0}\right\|_{H^{s+2 p-2}} .
\end{aligned}
$$

Again from (3.3), we know that

$\left\|H_{t}(t, \Lambda) u_{1}\right\|_{H^{s}}^{2} \leq C\left\|u_{1}\right\|_{H^{s}}^{2}, \quad \forall u_{1} \in S\left(\square^{n}\right), t>0$.

which implies that

$$
\begin{aligned}
& \int_{0}^{t}\left\|\int_{0}^{\tau} k(\sigma) H(\tau-\sigma, \Lambda)\left(-\Delta_{g}\right)^{p}\left(1-\Delta_{g}\right)^{-1} u_{0} d \sigma\right\|_{H^{s}}^{2} d \tau \\
& \leq\|k\|_{L^{2}}\left(\int_{0}^{t} k(\sigma)\left\|H(\tau-\sigma, \Lambda)\left(-\Delta_{g}\right)^{p}\left(1-\Delta_{g}\right)^{-1} u_{0}\right\|_{H^{s}}^{2} d \sigma\right) d \tau \\
& \leq\|k\|_{L^{2}}^{2} \int_{0}^{t}\left\|H(\tau-\sigma, \Lambda)\left(-\Delta_{g}\right)^{p}\left(1-\Delta_{g}\right)^{-1} u_{0}\right\|_{H^{s}}^{2} d \tau \\
& \leq C\left\|u_{0}\right\|_{H^{s+2 p-2}}^{2}
\end{aligned}
$$

where in the first inequality, we used the Jensen's inequality, while in the second inequality, we used the $L^{1}$-estimates for the convolution operation with respect to time (or changing the order of integration).

In a similar way, by (3.3), we have 


$$
\begin{aligned}
& \int_{0}^{t}\left\|\int_{0}^{\tau} k(\sigma) H_{t}(\tau-\sigma, \Lambda)\left(-\Delta_{g}\right)^{p}\left(1-\Delta_{g}\right)^{-1} u_{0} d \sigma\right\|_{H^{s-1}}^{2} d \tau \\
& \leq\|k\|_{L^{1}}\left(\int_{0}^{t} k(\sigma)\left\|H_{t}(\tau-\sigma, \Lambda)\left(-\Delta_{g}\right)^{p}\left(1-\Delta_{g}\right)^{-1} u_{0}\right\|_{H^{s-1}}^{2} d \sigma\right) d \tau \\
& \leq\|k\|_{L^{1}}^{2} \int_{0}^{t}\left\|H_{t}(\tau-\sigma, \Lambda)\left(-\Delta_{g}\right)^{p}\left(1-\Delta_{g}\right)^{-1} u_{0}\right\|_{H^{s-1}}^{2} d \tau \\
& \leq C\left\|u_{0}\right\|_{H^{s+2 p-2}}^{2}
\end{aligned}
$$

Thus, in term of (2.4) and the estimates of (3.2)-(3.8), as well as the fact that

$$
\begin{aligned}
u_{t}(t) & =G_{t}(t, \Lambda) u_{0}+H_{t}(t, \Lambda) u_{1} \\
& +\int_{0}^{t} H_{t}(t-\tau, \Lambda)\left(1-\Delta_{g}\right)^{-1} F(\tau) d \tau
\end{aligned}
$$

with $F(t, x)=k(t)\left(-\Delta_{g}\right)^{p} u_{0}(x)$ defined in (2.1), we have

$$
\begin{aligned}
\|u(t)\|_{H^{s+1}}^{2}+ & \left\|u_{t}(t)\right\|_{H^{s}}^{2} \\
& +\int_{0}^{t}\left(\|u(\tau)\|_{H^{s}}^{2}+\left\|u_{t}(\tau)\right\|_{H^{s-1}}^{2}\right) d \tau \leq C I_{0}^{2} .
\end{aligned}
$$

That is the conclusion of the theorem.

In order to prove Theorem 1.2, we need the following lemma which is a direct result of Proposition 1.

Lemma 3.1. With $\rho(\lambda)=\langle\lambda\rangle^{-2}$ introduced in Proposition $1, G(t, \lambda)$ and $H(t, \lambda)$ satisfy the following estimates:

(1). $|G(t, \lambda)| \leq C e^{-c \rho(\lambda) t}$

(2). $\left|G_{t}(t, \lambda)\right| \leq C e^{-c \rho(\lambda) t}\langle\lambda\rangle$,

(3). $|H(t, \lambda)| \leq C e^{-c \rho(\lambda) t}\langle\lambda\rangle^{-1}$,

(1). $\left|H_{t}(t, \lambda)\right| \leq C e^{-c \rho(\lambda) t}$.

By the above lemma, we have the following estimates:

Lemma 3.2. Let $r \geq 0, v \geq 0$ be real numbers, then the following estimates hold:

(1). $\|G(t, \Lambda) \varphi\|_{H^{r}} \leq C(1+t)^{-\frac{v}{2}}\|\varphi\|_{H^{r+\nu}}, \forall \varphi \in S\left(\square^{n}\right)$,

(2). $\left\|G_{t}(t, \Lambda) \varphi\right\|_{H^{r}} \leq C(1+t)^{-\frac{v}{2}}\|\varphi\|_{H^{r+v+1}}, \forall \varphi \in S\left(\square^{n}\right)$,

(3). $\|H(t, \Lambda) \varphi\|_{H^{r}} \leq C(1+t)^{-\frac{v}{2}}\|\varphi\|_{H^{r+v-1}}, \forall \varphi \in S\left(\square^{n}\right)$,

(4). $\left\|H_{t}(t, \Lambda) \varphi\right\|_{H^{r}} \leq C(1+t)^{-\frac{v}{2}}\|\varphi\|_{H^{r+\nu}}, \forall \varphi \in S\left(\square^{n}\right)$.
Proof. We only prove the case 1), but the other cases can be deduced similarly. In view of Lemma 3.11$)$ and the functional calculus (2.5) as well as the equivalence (1.2), we have that

$$
\begin{aligned}
\|G(t, \Lambda) \phi\|_{H^{r}}^{2} \leq & C \int_{0}^{\infty}\langle\lambda\rangle^{2 r}|G(t, \lambda)| d\left(P_{\lambda} \phi, \phi\right)_{L^{2}} \\
\leq & C \int_{0}^{\infty}\langle\lambda\rangle^{2 r} e^{-c p(\lambda) t} d\left(P_{\lambda} \phi, \phi\right)_{L^{2}} \\
= & C \int_{0}^{1}\langle\lambda\rangle^{2 r} e^{-c p(\lambda) t} d\left(P_{\lambda} \phi, \phi\right)_{L^{2}} \\
& +C \int_{0}^{\infty}\langle\lambda\rangle^{2 r} e^{-c p(\lambda) t} d\left(P_{\lambda} \phi, \phi\right)_{L^{2}} \\
= & : I_{1}+I_{2} .
\end{aligned}
$$

It is obvious that

$$
I_{1} \leq C e^{-c t}\|\phi\|_{L^{2}}^{2} .
$$

On the other hand,

$$
\begin{aligned}
I_{2} & \leq C(1+t)^{-v} \int_{0}^{\infty}\langle\lambda\rangle^{2(r+v)} d\left(P_{\lambda} \varphi, \varphi\right)_{L^{2}} \\
& \leq C(1+t)^{-v}\|\varphi\|_{H^{r+v}}^{2} .
\end{aligned}
$$

Here

$$
r \geq 0, v \geq 0, r+v \leq s+\max \{1,2 p-2\} .
$$

Thus the result for the case 1) is proved.

Proof of Theorem 1.2. Let $r \geq 0$, then from (2.4) we have that

$$
\begin{aligned}
& \|u(t)\|_{H^{r}} \leq\left\|G(t, \Lambda) u_{0}\right\|_{H^{r}}+\left\|H(t, \Lambda) u_{1}\right\|_{H^{r}} \\
& +\left\|\int_{0}^{t} k(\tau) H(t-\tau, \Lambda)\left(-\Delta_{g}\right)^{p}\left(1-\Delta_{g}\right)^{-1} u_{0} d_{\tau}\right\|_{H^{r}} \\
& \quad=I+I I+I I I .
\end{aligned}
$$

By Lemma 3.2, we know that

$$
I+I I \leq C(1+t)^{-\frac{v_{1}}{2}}\left\|u_{0}\right\|_{H^{r+v_{1}}}+C(1+t)^{-\frac{v_{2}}{2}}\left\|u_{1}\right\|_{H^{r+v_{2}-1}} .
$$

And

$$
\begin{aligned}
& I I I \leq \int_{0}^{\frac{t}{2}} k(\tau)\left\|H(t-\tau, \Lambda)\left(-\Delta_{g}\right)^{p}\left(1-\Delta_{g}\right)^{-1} u_{0}\right\|_{H^{r}} d \tau \\
& +\int_{\frac{t}{2}}^{t} k(\tau)\left\|H(t-\tau, \Lambda)\left(-\Delta_{g}\right)^{p}\left(1-\Delta_{g}\right)^{-1} u_{0}\right\|_{H^{r}} d \tau \\
& \leq C(1+t)^{-\frac{v_{3}}{2}}\left\|\left(-\Delta_{g}\right)^{p}\left(1-\Delta_{g}\right)^{-1} u_{0}\right\|_{H^{r+v_{3}-1}} \int_{0}^{\frac{t}{2}} k(\tau) d \tau \\
& +C e^{-c t}\left\|\left(-\Delta_{g}\right)^{p}\left(1-\Delta_{g}\right)^{-1} u_{0}\right\|_{H^{r+v_{3}-1}} \\
& \leq C(1+t)^{-\frac{v_{3}}{2}}\left\|u_{0}\right\|_{H^{r+v_{3}+2 p-3}},
\end{aligned}
$$

where in the second step, we used the exponentially decay 
property of $k(t)$, which is a direct result from the Assumption [A]. Thus, we have

$$
\begin{aligned}
\|u(t)\|_{H^{r}} \leq C(1+t)^{-\frac{v_{1}}{2}}\left\|u_{0}\right\|_{H^{r+\left(v_{1}-\max \{1,2 p-2\}+1\right)-1+\max \{1,2 p-2\}}} \\
+C(1+t)^{-\frac{v_{2}}{2}}\left\|u_{1}\right\|_{H^{r+v_{2}-1}} \\
+(1+t)^{-\frac{v_{3}}{2}}\left\|u_{0}\right\|_{H^{r+\left(v_{3}+2 p-2-\max \{1,2 p-2\}+1\right)-1+\max \{1,2 p-2\}}} .
\end{aligned}
$$

Here $v_{1} \geq 0, v_{2} \geq 0, v_{3} \geq 0$ satisfy

$$
\left\{\begin{array}{l}
r+v_{1} \leq s+\max \{1,2 p-2\} \\
r+v_{2}-1 \leq s \\
r+v_{3}+2 p-3 \leq s+\max \{1,2 p-2\} .
\end{array}\right.
$$

Choose the smallest real numbers $v_{1}, v_{2}, v_{3}$ such that

$$
\left\{\begin{array}{l}
v_{1}-\max \{1,2 p-2\}+1 \geq \sigma, \\
v_{2} \geq \sigma, \\
v_{3}+2 p-2-\max \{1,2 p-2\} \geq \sigma .
\end{array}\right.
$$

It gives that

$$
\left\{\begin{array}{l}
v_{2}=\sigma, \\
v_{1}=v_{2}+\max \{1,2 p-2\}-1, \\
v_{3}=v_{2}+\max \{1,2 p-2\}-(2 p-2) .
\end{array}\right.
$$

Thus, the inequality (3.10) holds with $r$ satisfying

$$
0 \leq r \leq s+1-\sigma \text {. }
$$

Taking the maximal $r$, i.e., $r=s+1-\sigma$, we obtain

$$
\|u(t)\|_{H^{s+1-\sigma}} \leq C I_{0}(1+t)^{-\frac{\sigma}{2}} .
$$

That is the result for $u(t)$.

The estimate for $u_{t}(t)$ can be proved in a similar way by just using the fact (3.9) and Lemma 3.2, and we omit the details.

\section{Global existence and decay estimates of solutions to the semilinear problem.}

In this section, by virtue of the properties of solution operators, we prove the global existence and optimal decay estimates of solutions to the semilinear problem by employing the contraction mapping theorem.

From (2.1), we know that the solution to (1.1) can be expressed as

$$
\begin{aligned}
& u(t)=G(t, \Lambda) u_{0}+H(t, \Lambda) u_{1} \\
& +\int_{0}^{t} H(t-\tau, \Lambda) k(\tau)\left(1-\Delta_{g}\right)^{-1}\left(-\Delta_{g}\right)^{p} u_{0} d \tau \\
& +\int_{0}^{t} H(t-\tau, \Lambda)\left(1-\Delta_{g}\right)^{-1} f\left(u(\tau), u_{t}(\tau), \nabla u(\tau)\right) d \tau .
\end{aligned}
$$

Lemma 4.1 (Moser estimates). Assume that $r \geq 0$ be a real number, then

$$
\|u v\|_{H^{r}} \leq C\left(\|u\|_{L^{\infty}}\|v\|_{H^{r}}+\|v\|_{L^{\infty}}\|u\|_{H^{r}}\right) .
$$

By the previous lemma and an inductive argument, we have the following estimates:

Lemma 4.2. Assume that $\alpha \geq 1, \beta \geq 1$ be integers, and $r \geq 0$ be a real number, then

$$
\begin{array}{r}
\left\|u^{\alpha} v^{\beta}\right\|_{H^{r}} \leq C\left(\| u \| _ { L ^ { \infty } } ^ { \alpha - 1 } \| v \| _ { L ^ { \infty } } ^ { \beta - 1 } \left(\|u\|_{L^{\infty}}\|v\|_{H^{r}}\right.\right. \\
\left.+\|v\|_{L^{\infty}}\|u\|_{H^{r}}\right) .
\end{array}
$$

Define

$$
\begin{aligned}
X:= & \left\{u \in C\left([0, \infty), H^{s+1}\left(\square^{n}\right)\right) \bigcap\right. \\
& \left.C^{1}\left([0, \infty), H^{s}\left(\square^{n}\right)\right) ;\|u\|<+\infty\right\},
\end{aligned}
$$

here

$$
\begin{aligned}
\|u\|_{X} & :=\sup _{0 \leq \sigma \leq s+1} \sup _{t \geq 0}\left\{(1+t)^{\frac{\sigma}{2}}\|u(t)\|_{H^{s+1-\sigma}}\right\} \\
& +\sup _{0 \leq \sigma \leq s+1} \sup _{t \geq 0}\left\{(1+t)^{\frac{\sigma}{2}}\left\|u_{t}(t)\right\|_{H^{s-\sigma}}\right\} .
\end{aligned}
$$

Proposition 2. There exists $C>0$ such that

$$
\|U(t)\|_{L^{\infty}} \leq C\|u\|_{X} .
$$

Proof. By the Sobolev imbedding theorems, we have

$$
\|U(t)\|_{L^{\infty}} \leq C\|U(t)\|_{H^{s}} .
$$

By the definition of $\|u\|_{X}$ and $\|\nabla u\|_{H^{s}} \leq C\|u\|_{H^{s+1}}$, we obtained the result.

Denote

$B_{R}:=\left\{u \in X ;\|u\|_{X} \leq R\right\}$,

$U:=\left(u, u_{t}, \nabla u\right)$,

$\Phi[u](t):=\Phi_{0}(t)+\int_{0}^{t} H(t-\tau, \Lambda)\left(1-\Delta_{g}\right)^{-1} f(U) d \tau$,

$\Phi_{0}(t):=G(t, \Lambda) u_{0}+H(t, \Lambda) u_{1}$

$+\int_{0}^{t} H(t-\tau, \Lambda) k(\tau)\left(1-\Delta_{g}\right)^{-1}\left(-\Delta_{g}\right)^{p} u_{0} d \tau$.

We will prove that $u \rightarrow \Phi(u)$ is a contraction mapping on $B_{R}$ for some $R>0$. 
Proof of Theorem 1.3. We denote

$V:=\left(v, v_{t}, \nabla v\right), W:=\left(w, w_{t}, \nabla w\right)$,then

$\Phi[v](t)-\Phi[w](t)=$

$\int_{0}^{t} H(t-\tau, \Lambda)\left(1-\Delta_{g}\right)^{-1}(f(V)-f(W))(\tau) d \tau$.

Step 1: We prove:

$\|\Phi[v](t)-\Phi[w](t)\|_{H^{s+1-\sigma}} \leq$

$$
C(1+t)^{-\frac{\sigma}{2}}\|(v, w)\|_{X}^{\eta-1}\|v-w\|_{X}
$$

and $0 \leq \sigma \leq s+1$

Indeed,

$\|(\Phi[v]-\Phi[w])(t)\|_{H^{s+1-\sigma}}$

$\leq\left(\int_{0}^{\frac{t}{2}}+\int_{\frac{t}{2}}^{t}\right)$

$\left\|H(t-\tau, \Lambda)\left(1-\Delta_{g}\right)^{-1}(f(V)-f(W))(\tau)\right\|_{H^{s+1-\sigma}} d \tau$

$\leq\left(\int_{0}^{\frac{t}{2}}+\int_{\frac{t}{2}}^{t}\right)$

$\left\|H(t-\tau, \Lambda)\left(1-\Delta_{g}\right)^{-1}(f(V)-f(W))(\tau)\right\|_{H^{s+1-\sigma-2}} d \tau$

$=: I_{1}+I_{2}$.

In view of lemma 3.23 ), we have that

$I_{1} \leq$

$C \int_{0}^{\frac{t}{2}}(1+t-\tau)^{-\frac{v}{2}}\|(f(V)-f(W))(\tau)\|_{H^{s+1-\sigma-2+v-1}} d \tau$

$\leq C \int_{0}^{\frac{t}{2}}(1+t-\tau)^{-\frac{v}{2}}\|(f(V)-f(W))(\tau)\|_{H^{s+1-(\sigma-v-3)}} d \tau$

By Lemma 4.2, we get that

$\|(f(V)-f(W))(\tau)\|_{H^{s+1-(\sigma-(v-3)]}} \leq C\|(V, W)(\tau)\|_{L^{\infty}}^{\eta-2}$

$\times\left\{\|(V, W)(\tau)\|_{L^{\infty}}\|(V-W)(\tau)\|_{H^{s+1-[\sigma-(v-3)]}}\right.$

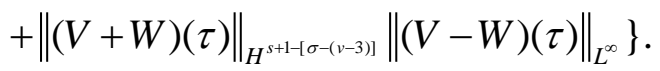

In view of (4.1), we have that

$\|(f(V)-f(W))(\tau)\|_{H^{s+1-[\sigma-(v-3)]}} \leq$

$$
C(1+\tau)^{-\frac{\sigma-(v-3)}{2}}\|(v, w)\|_{X}^{\eta-1}\|(v, w)\|_{X} d \tau(4.5)
$$

Let $v=\sigma$ in (4.5), we have that

$$
\begin{aligned}
& I_{1} \leq \\
& C \int_{0}^{\frac{t}{2}}(1+t-\tau)^{-\frac{v}{2}}(1+\tau)^{-\frac{\sigma-(v-3)}{2}}\|(v, w)\|_{X}^{\eta-1}\|(v-w)\|_{X} d \tau \\
& \leq C \int_{\frac{t}{2}}^{t}\left(1+\frac{t}{2}\right)^{-\frac{\sigma}{2}} \int_{0}^{\frac{t}{2}}(1+\tau)^{-\frac{-3}{2}}\|(v, w)\|_{X}^{\eta-1}\|(v-w)\|_{X} d \tau \\
& \leq C(1+t)^{-\frac{\sigma}{2}}\|(v, w)\|_{X}^{\eta-1}\|(v-w)\|_{X} \cdot \quad \quad(4.6) \\
& \text { Le } v=0 \text { in }(4.5), \text { we have that } \\
& I_{2} \leq \\
& C \int_{\frac{t}{2}}^{t}(1+t-\tau)^{-\frac{v}{2}}(1+\tau)^{-\frac{\sigma-(v-3)}{2}}\|(v, w)\|_{X}^{\eta-1}\|(v-w)\|_{X} d \tau \\
& \leq C \int_{\frac{t}{2}}^{t}(1+t)^{-\frac{\sigma+3}{2}}\|(v, w)\|_{X}^{\eta-1}\|(v-w)\|_{X} d \tau \\
& \leq C \int_{\frac{t}{2}}^{t}\left(1+\frac{t}{2}\right)^{-\frac{\sigma}{2}}(1+\tau)^{-\frac{3}{2}}\|(v, w)\|_{X}^{\eta-1}\|(v-w)\|_{X} d \tau \\
& \leq C(1+t)^{-\frac{\sigma}{2}}\|(v, w)\|_{X}^{\eta-1}\|(v-w)\|_{X} . \quad(4.7)
\end{aligned}
$$

By virtue of (4.6) and (4.7), we obtain the desired results.

Step 2. we prove:

$$
\begin{aligned}
& \left\|\left(\Phi_{t}[v]-\Phi_{t}[w]\right)(t)\right\|_{H^{s-\sigma}} \leq \\
& C(1+t)^{-\frac{\sigma}{2}}\|(v, w)\|_{X}^{\eta-1}\|(v-w)\|_{X} .
\end{aligned}
$$

Indeed,

$\left\|\left(\Phi_{t}[v]-\Phi_{t}[w]\right)(t)\right\|_{H^{s-\sigma}}$

$\leq\left(\int_{0}^{\frac{t}{2}}+\int_{\frac{t}{2}}^{t}\right)$

$\left\|H_{t}(t-\tau, \Lambda)\left(1-\Delta_{g}\right)^{-1}(f(V)-f(W))(\tau)\right\|_{H^{s-\sigma}} d \tau$

$\leq\left(\int_{0}^{\frac{t}{2}}+\int_{\frac{t}{2}}^{t}\right)$

$\left\|H_{t}(t-\tau, \Lambda)(f(V)-f(W))(\tau)\right\|_{H^{s-\sigma-2}} d \tau$

$=: I_{3}+I_{4}$.

In view of Lemma 3.24 ), we have

$I_{3} \leq C \int_{0}^{\frac{t}{2}}(1+t-\tau)^{-\frac{v}{2}}\|(f(V)-f(W))(\tau)\|_{H^{s+v-\sigma-2}} d \tau$

$=C \int_{0}^{\frac{t}{2}}(1+t-\tau)^{-\frac{v}{2}}\|(f(V)-f(W))(\tau)\|_{H^{s+1-(\sigma-(v-3))}} d \tau$.

In a similar way to (4.6) and (4.7), we have 
$I_{3} \leq$

$\left.C \int_{0}^{\frac{t}{2}}(1+t-\tau)^{-\frac{v}{2}}(1+\tau)\right)^{-\frac{\sigma-(v-3)}{2}}\|(v, w)\|_{X}^{\eta-1}\|(v-w)\|_{X} d \tau$

$\leq C \int_{\frac{t}{2}}^{t}\left(1+\frac{t}{2}\right)^{-\frac{\sigma}{2}} \int_{0}^{\frac{t}{2}}(1+\tau)^{-\frac{-3}{2}}\|(v, w)\|_{X}^{\eta-1}\|(v-w)\|_{X} d \tau$

$\leq C(1+t)^{-\frac{\sigma}{2}}\|(v, w)\|_{X}^{\eta-1}\|(v-w)\|_{X}$.

and

$$
\begin{aligned}
& I_{4} \leq \\
& C \int_{\frac{t}{2}}^{t}(1+t-\tau)^{-\frac{v}{2}}(1+\tau)^{-\frac{\sigma-(v-3)}{2}}\|(v, w)\|_{X}^{\eta-1}\|(v-w)\|_{X} d \tau \\
& \leq C \int_{\frac{t}{2}}^{t}(1+t)^{-\frac{\sigma+3}{2}}\|(v, w)\|_{X}^{\eta-1}\|(v-w)\|_{X} d \tau \\
& \leq C \int_{\frac{t}{2}}^{t}\left(1+\frac{t}{2}\right)^{-\frac{\sigma}{2}}(1+\tau)^{-\frac{3}{2}}\|(v, w)\|_{X}^{\eta-1}\|(v-w)\|_{X} d \tau \\
& \leq C(1+t)^{-\frac{\sigma}{2}}\|(v, w)\|_{X}^{\eta-1}\|(v-w)\|_{X} .
\end{aligned}
$$

By virtue of (4.9) and (4.10), we obtain the desired results.

Combining the estimates (4.2) and (4.8), we obtain that

$$
\|\Phi[v]-\Phi[w]\|_{X} \leq C\|(v, w)\|_{X}^{\eta-1}\|v-w\|_{X} .
$$

So far we proved that

$\|\Phi[v]-\Phi[w]\|_{X} \leq C_{1} R^{\eta-1}\|v-w\|_{X}$ if $v, w \in B_{R}$.

On the other hand, $\Phi(0)(t)=\Phi_{0}(t)$, and from Theorem 1.2 we know that $\|\Phi[0]\|_{X} \leq C_{2} I_{0}$ if $I_{0}$ is suitably small.Take $R=2 C_{2} I_{0}$. if $I_{0}$ is suitably small such that

$C_{1} R^{\eta-1} \leq \frac{1}{2}$, then we have that

$$
\|\Phi[v]-\Phi[w]\|_{X} \leq \frac{1}{2}\|v-w\|_{X} .
$$

It yields that, for $v \in B_{R}$,

$$
\|\Phi[v]\|_{X} \leq\|\Phi[0]\|_{X}+\frac{1}{2}\|v\|_{X} \leq C_{2} I_{0}+\frac{1}{2} R=R
$$

Thus $\Phi[v] \in B_{R}, v \rightarrow \Phi[v]$ is a contraction mapping on $B_{R}$.and by the fixed point theorem there exists a unique $u \in B_{R}$ satisfying $\Phi[u]=u$, and it is the solution to the semilinear problem (1.1) satisfying the decay estimates (1.4) and (1.5). So far we complete the proof of Theorem 1.3.

\section{REFERENCES}

[1] M. E. Bradley and S. Lenhart, Bilinear spatial control of the velocity term in a Kirchhoff plate equation, Electronic J. Differential Equations, 2001 (2001), 1-15.

[2] C. Buriol, Energy decay rates for the Timoshenko system of thermoelastic plates,Nonlinear Analysis, 64 (2006), 92-108.

[3] R. C. Charão, E. Bisognin, V. Bisognin and A.F. Pazoto, Asymptotic behavior for a dissipative plate equation in $\mathrm{R}$ $\mathrm{N}$ with periodic coefficients, Electronic J. Differential Equations, 2008 (2008), 1-23.

[4] C. R. da Luz and R. C. Charão, Asymptotic properties for a semi-linear plate equation in unbounded domains, J. Hyperbolic Differential Equations, 6 (2009), 269-294.

[5] M. Dimassi and J. Sjöstrand, Spectral Asymptotics in the Semi-Classical Limit, London Mathematical Society Lecture Note Series, 268, Cambridge University Press, (1999).

[6] P. M. N. Dharmawardane, J. E. Muñoz Rivera and S. Kawashima, Decay property for second order hyperbolic systems of viscoelastic materials, J. Math. Anal. Appl., 366(2010), 621-635.

[7] Y. Enomoto, On a thermoelastic plate equation in an exterior domain, Math. Meth.Appl. Sci., 25 (2002), 443 472 .

[8] L. Hörmander, Analysis of Linear Partial Differential Operators, Vol. III, Springer-Verlag, (1983).

[9] T. Hosono and S. Kawashima, Decay property of regularity-loss type and application to some nonlinear hyperbolic-elliptic system, Math. Models Meth. Appl. Sci., 16 (2006),1839-1859.

[10] K. Ide and S. Kawashima, Decay property of regularityloss type and nonlinear effects for dissipative Timoshenko system, Math. Models Meth. Appl. Sci., 18 (2008), 1001-1025.

[11] H. J. Lee, Uniform decay for solution of the plate equation with a boundary condition of memory type, Trends in Math, 9 (2006), 51-55.

[12] Y. Liu, Decay of solutions to an inertial model for a semilinear plate equation with memory, J. Math. Anal. Appl., 394 (2012), 616-632.

[13] [Y. Liu and S. Kawashima, Global existence and asymptotic behavior of solutions for quasi-linear dissipative plate equation, Discrete Contin. Dyn. Syst., 29 (2011), 1113-1139.

[14] [Y. Liu and S. Kawashima, Global existence and decay of solutions for a quasi-linear dissipative plate equation, J. Hyperbolic Differential Equations, 8 (2011), 591-614.

[15] Y. Liu and S. Kawashima, Decay property for a plate equation with memory-type dissipation, Kinet. Relat. Mod., 4 (2011), 531-547.

[16] S. Mao and Y. Liu, Decay of solutions to generalized plate type equations with memory,Kinet. Relat. Mod., 7 (2014), 121-131.

[17] S. Mao and Y. Liu, Decay properties for solutions to plate type equations with variable coefficients, Kinet. Relat. Mod., 10 (2017), 785-797.16 
[18] N. Mori and S. Kawashima, Decay property for the Timoshenko system with Fourier's type heat conduction, J. Hyperbolic Differential Equations, 11 (2014), 135-157.

[19] N. Mori and S. Kawashima, Decay property of the Timoshenko-Cattaneo system, Anal.Appl., (2015), 1-21.

[20] N. Mori, J. Xu and S. Kawashima, Global existence and optimal decay rates for the Timoshenko system: The case of equal wave speeds, J. Differ. Equations., 258 (2015),1494-1518.

[21] M. Reed, and B. Simon, Methods of Modern Mathematical Physics, Vol. I, Academic Press, New York, (1975).

[22] J. E. Mũ noz Rivera, M.G. Naso and F.M. Vegni, Asymptotic behavior of the energy for a class of weakly dissipative second-order systems with memory, J. Math. Anal. Appl.,286 (2003), 692-704.

[23] Y. Sugitani and S. Kawashima, Decay estimates of solutions to a semi-linear dissipative plate equation, J. Hyperbolic Differential Equations, 7 (2010), 471-501.

[24] J. Xu, N. Mori and S. Kawashima, Global existence and minimal decay regularity for the Timoshenko system: The case of non-equal wave speeds, J. Differ. Equations., 259(2015), 5533-5553. 